\title{
BMJ Open Protocol for a 1-year prospective, longitudinal cohort study of patients undergoing Roux-en-Y gastric bypass and sleeve gastrectomy: the BARI- LIFESTYLE observational study
}

Friedrich C Jassil, ${ }^{1,2}$ Alisia Carnemolla, ${ }^{1,3}$ Helen Kingett, ${ }^{2,3}$ Bruce Paton, ${ }^{4}$ Aidan G O'Keeffe, ${ }^{5}$ Jacqueline Doyle, ${ }^{2,3}$ Stephen Morris, ${ }^{6}$ Neville Lewis, ${ }^{7}$ Amy Kirk, ${ }^{2,3}$ Andrea Pucci, ${ }^{1,2}$ Kusuma Chaiyasoot, ${ }^{1,2}$ Rachel L Batterham ${ }^{1,2,3}$

To cite: Jassil FC, Carnemolla A, Kingett $\mathrm{H}$, et al. Protocol for a 1-year prospective, longitudinal cohort study of patients undergoing Roux-en-Y gastric bypass and sleeve gastrectomy: the BARI-LIFESTYLE observational study. BMJ Open 2018;8:e020659. doi:10.1136/ bmjopen-2017-020659

- Prepublication history for this paper is available online. To view these files, please visit the journal online (http://dx.doi. org/10.1136/bmjopen-2017020659).

Received 15 November 2017 Revised 5 January 2018 Accepted 31 January 2018

Check for updates

For numbered affiliations see end of article.

Correspondence to

Professor Rachel L Batterham; r.batterham@ucl.ac.uk

\section{ABSTRACT}

Introduction Roux-en- $Y$ gastric bypass and sleeve gastrectomy are the two most common bariatric surgery performed in the UK that result in comparable weight loss and remission of obesity-associated comorbidities. However, there is a paucity of studies examining the impact of these procedures on body composition, physical activity levels, sedentary behaviour, physical function and strength, dietary intake, health-related quality of life and costs.

Methods and analysis The BARI-LIFESTYLE observational study is a 1-year prospective, longitudinal cohort study within a real-world routine clinical care setting aiming to recruit 100 patients with severe obesity undergoing either primary RouX-en- $Y$ gastric bypass or sleeve gastrectomy from two bariatric centres in London, UK. Participants will be followed up four times during the study period; presurgery baseline (T0) and at 3 (T1), 6 (T2) and 12 months (T3) postsurgery. In addition to the standard follow-up investigations, assessments including dual-energy X-ray absorptiometry scan, bioelectric impedance analysis, 6 min walk test, sit-to-stand test and handgrip test will be undertaken together with completion of questionnaires. Physical activity levels and sedentary behaviour will be assessed using accelerometer, and dietary intake will be recorded using a 3-day food diary. Outcome measures will include body weight, body fat mass, lean muscle mass, bone mineral density, physical activity levels, sedentary behaviour, physical function and strength, dietary intake, health-related quality of life, remission of comorbidities, healthcare resource utilisation and costs.

Ethics and dissemination This study has been reviewed and given a favourable ethical opinion by London-Dulwich Research Ethics Committee (17/ L0/0950). The results will be presented to stakeholder groups locally, nationally and internationally and published in peer-reviewed medical journals. The layperson summary of the findings will be published on the Centre for Obesity Research, University College London website (http://www.ucl.ac.uk/obesity).

\section{Strengths and limitations of this study}

- A comprehensive prospective, longitudinal study with detailed assessments undertaken prior to and for 1 year following bariatric surgery examining changes in body composition, physical activity (PA) levels, sedentary behaviour, physical function and strength, dietary intake, health-related quality of life and costs, relative to baseline presurgery.

- The use of validated research tools (accelerometer to assess PA levels and sedentary behaviour, dual-energy X-ray absorptiometry (DXA) scan to assess body composition and validated questionnaires) will generate high-quality data.

- The study design does not include a conventional intensive lifestyle intervention (non-surgical) as a comparator group and patients will not be randomised to Roux-en-Y gastric bypass (RYGB) or sleeve gastrectomy (SG) in order to reflect current real-world clinical care.

- A potential sample selection bias due to exclusion of patients with functional limitation and/or non-ambulatory and patients with more than $200 \mathrm{~kg}$ of body weight owing to the weight limit of the DXA scan.

- A relatively small sample size, nevertheless, this number is adequate to generate indepth insights into the various outcomes of RYGB and SG as delivered in the UK healthcare setting.

\section{INTRODUCTION}

Bariatric surgery engenders marked sustained weight loss and is recommended by the National Institute for Health and Care Excellence (NICE) as a treatment option for people of severe obesity, ${ }^{1}$ estimated to affect approximately 2.6 million adults in the UK. ${ }^{2}$ Roux-en-Y gastric bypass (RYGB) and sleeve gastrectomy (SG) are now the two most common procedures performed in the UK, which result in comparable weight loss and 
remission of obesity-associated comorbidities. ${ }^{3}$ However, there is a paucity of studies examining the impact of these procedures on body composition, particularly bone mineral density (BMD), physical activity (PA) levels, sedentary behaviour, physical function and strength, dietary intake, health-related quality of life (HRQoL) and costs. Furthermore, current eligibility and success criteria of bariatric surgery are mainly based on body weight, body mass index and excess weight loss but evidence have shown various beneficial outcomes of the surgery above and beyond weight loss alone, hence highlighting the need for more functional preoperative and postoperative patient assessment. ${ }^{45}$

Bariatric surgery leads to a marked decrease in fat mass (FM), but fat free mass (FFM) particularly bone mass is also reduced postsurgery, ${ }^{6}$ potentially negatively impacting on physical function and strength, and putting patients at increased risk of osteoporotic fracture in the future. ${ }^{78}$ Moreover, a recent study has revealed a positive association between changes in adiposity with cardiometabolic outcomes postsurgery, indicating the usefulness of incorporating body composition assessment. ${ }^{9}$ Surgical modification of the gastrointestinal tract impairs the intake and/or absorption of essential nutrients for bone health that consequently perturbs bone metabolism, leading to BMD deterioration. ${ }^{781011}$ Significant bone mass loss has been reported to occur rapidly in the first year of surgery and continues to deteriorate up to 3 years even after maximum weight loss has been achieved. ${ }^{10}$ However, these data are mainly based on studies undertaken in patients who underwent RYGB whereas SG is now the most common procedure undertaken both in the UK and globally. ${ }^{3}{ }^{12}$ Currently, it is unclear whether the rate of bone mass loss following SG parallels weight loss. ${ }^{13-15}$ Given that the number of younger patients and women of childbearing age undergoing bariatric surgery continues to increase and BMD measurement is not a routine follow-up investigation, ${ }^{16}$ there is an urgent need to assess the impact of RYGB and SG on bone health in the UK bariatric population.

Adherence to a postbariatric lifestyle changes is the cornerstone of a successful weight loss. ${ }^{17}$ Studies have shown that greater PA, lower sedentary time and high compliance to dietary recommendation postsurgery associate with greater weight loss and FM loss, preservation of lean muscle mass (LMM) and bone mass, as well as improvement in HRQoL. ${ }^{18-22}$ However, patients spend $80 \%$ of their waking time in sedentary behaviour postsurgery, ${ }^{23}$ activity that associates with increased risk of cardiometabolic disease and mortality. ${ }^{24}$ Following surgery, patients are advised to undertake at least 150 min of moderate-to-vigorous physical activity (MVPA) per week, a duration and intensity that are recommended to reap the metabolic benefit of PA. ${ }^{25}$ However, objectively measured MVPA decreases postsurgery with only $10 \%$ of patients achieving the recommended MVPA levels. ${ }^{26}$ Likewise, a recent study undertaken in the UK has reported that weight loss postsurgery did not correspond to improvement in MVPA and sedentary behaviour. However, the small sample size of this study $(n=22)$ together with relatively short follow-up period limited its generalisability. ${ }^{27}$ Further studies are therefore required to expand the information in this regard. In terms of dietary recommendations, daily protein intake of $60 \mathrm{~g}$ or more postsurgery is crucial for increasing satiety, preserving LMM, improving body composition and preventing against weight regain. ${ }^{28-31}$ However, most patients are unable to achieve this in the first postoperative year, the period when rapid weight loss occurs. ${ }^{32}$ Whether this is also the case for UK bariatric population is not known as no such data has ever been reported thus far. $^{32}$

Impaired HRQoL is common in obesity ${ }^{33}$ and often one of the driving factors for seeking weight loss surgery. ${ }^{34}$ HRQoL is defined as individuals' perception of well-being that refers to physical, psychological and social domains of health. ${ }^{35}$ Most studies reported improvement in all HRQoL domains with greater scores observed in the first postoperative year although some studies showed that the improvement is limited to only the physical domain but not the mental health component of HRQoL. ${ }^{36}$ Despite mounting evidence in the international literature reporting the beneficial impact of bariatric surgery on HRQoL, data from the UK bariatric population does not exist. ${ }^{37}$ There is some evidence that bariatric surgery can reduce in cost savings that offset the initial costs of surgery, though little UK evidence for RYGB and SG. ${ }^{38-40}$

Taken together the lack of postoperative data coupled with recommendations from systematic reviews ${ }^{26} 32$ provide a strong rational to undertake a prospective study to evaluate the impact of RYGB and SG on body composition particularly BMD, PA levels, sedentary behaviour, physical function and strength, dietary intake, HRQoL and costs in a UK bariatric population. Information gained from this study will provide valuable data to inform the implementation of future postsurgery lifestyle programmes with the aim of maximising the beneficial outcomes of bariatric surgery as highlighted by NICE. ${ }^{1}$ This paper details the study design and outcomes of the BARI-LIFESTYLE observational study.

\section{OBJECTIVES}

The overall objective of BARI-LIFESTYLE observational study is to evaluate the impact of RYGB and SG on changes in body weight, body composition, PA levels, sedentary behaviour, physical function and strength, dietary intake, HRQoL, remission of comorbidities, healthcare resource utilisation and costs in a cohort of 100 patients.

The specific objectives are to evaluate postsurgery changes in:

1. percentage weight loss (\%WL) at 1 year postsurgery, relative to baseline presurgery weight;

2. body FM, assessed using dual-energy X-ray absorptiometry (DXA) scan and bioelectrical impedance 
analysis (BIA), relative to presurgery at 12 months postsurgery;

3. LMM, assessed using DXA scan and BIA, relative to presurgery at 12 months postsurgery;

4. BMD, assessed using DXA scan and BIA, relative to presurgery at 12 months postsurgery;

5. PA levels (light, moderate, vigorous), percentage achieving $150 \mathrm{~min}$ of MVPA in a week and sedentary behaviour assessed using accelerometer at 3, 6 and 12 months postsurgery, relative to presurgery;

6. physical function and strength assessed using $6 \mathrm{~min}$ walk test (6MWT), sit-to-stand (STS) test and handgrip test at 3,6 and 12 months postsurgery, relative to presurgery;

7. dietary intake assessed using food diary at 3, 6 and 12 months postsurgery, relative to presurgery;

8. HRQoL assessed using EuroQol-5D-3L (EQ-5D3L) and Impact of Weight on Quality of Life-Lite (IWQOL-Lite) at 3, 6 and 12 months postsurgery, relative to presurgery;

9. characteristics of attitude and symptoms of depression assessed using Beck Depression Inventory-II (BDI-II) at 3, 6 and 12 months postsurgery, relative to presurgery;

10. obesity-associated comorbidities (type 2 diabetes (T2D), dyslipidaemia, hypertension, obstructive sleep apnoea (OSA)) at 3, 6 and 12 months postsurgery, relative to presurgery;

11. healthcare resource utilisation and costs assessed using an adapted version of the Client Service Receipt Inventory (CSRI) at 3, 6 and 12 months postsurgery, relative to presurgery.

\section{METHODS AND ANALYSIS}

\section{Study design and setting}

BARI-LIFESTYLE observational study is a prospective, longitudinal cohort study within routine clinical care setting of patients undergoing bariatric surgery conducted in London, UK (figure 1). A total of 100 patients who are planned to undergo either primary RYGB or SG will be recruited over a 2-year period from 2018 to 2019, and will be followed for up to 12 months postsurgery. Recruitment will take place at the Bariatric Centre for Weight Management and Metabolic Surgery, University College London Hospitals (UCLH) (study site) and the Bariatric and Obesity Surgery Clinic at the Whittington Hospital that acts as a participant identification centre (PIC). Participants recruited from the Whittington Hospital will have their surgical procedure undertaken at the same centre, but all study procedures such as written informed consent, baseline assessment, postsurgery follow-up care and study assessments will be undertaken by the bariatric team at UCLH. In both centres, the decision for procedure selection is based on informed patient preference after standardised counselling including details, potential risks, and benefits of each procedure that adheres to the current international

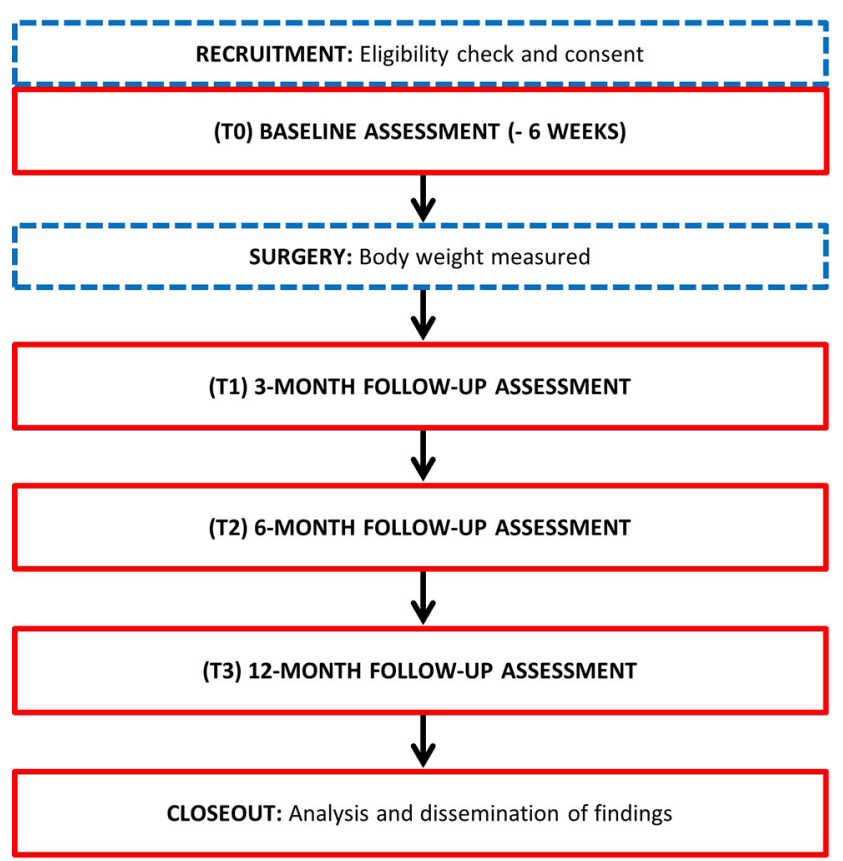

Figure 1 Flow diagram of participant enrolment, consent, assessment and associated timeline.

guideline for the surgical recommendation for obesity and weight-related disease. ${ }^{41}$ This study is carried out by the Centre for Obesity Research, Division of Medicine, University College London (UCL), with an expected total duration of 36 months, from the first participant enrolled to last participant follow-up.

\section{Participants and recruitment}

Patients will be screened for suitability for the study by the bariatric team at the study site and PIC based on the inclusion and exclusion criteria when they attend the standard presurgical assessment (box 1). Verbal consent will be sought from those fulfilling the eligibility criteria and interested in participating to be approached by a research investigator. Patients will be given a participant information sheet inviting them to participate in a 1-year prospective, longitudinal cohort study looking at the effect of bariatric surgery on body weight, body composition, PA levels, sedentary behaviour, physical function and strength, dietary intake, HRQoL, remission of comorbidities, healthcare resource utilisation and costs. Consented participants will then be scheduled to attend a baseline assessment, approximately 6 weeks before surgery day at the study site. Each participant will be given a Fitbit Alta HR to enable them to self-monitor their activity levels and to reduce sedentary behaviour. Based on the weekly number of bariatric procedures undertaken at UCLH and Whittington Hospital and after considering the eligibility criteria, estimated recruitment rate is approximately seven participants per month. Hence, the expected recruitment period for the study is $15-20$ months.

All participants will receive the standardised postbariatric care as stipulated by NICE. ${ }^{1}$ Participants will 
Box 1 Participant eligibility criteria for participation in the BARI-LIFESTYLE observational study

\section{Inclusion criteria}

- Adult aged between 18 and 65 years.

- Planned to undergo either primary RYGB or SG surgery and fulfilling NICE eligibility criteria for bariatric surgery. ${ }^{1}$

- Able to read and write in English.

- Willing and able to provide written informed consent.

- Able to comply with study protocol.

- Willing and able to wear a Fitbit wrist-based activity tracker device and an ActiGraph device.

\section{Exclusion criteria}

- More than $200 \mathrm{~kg}$ of body weight due to the limitation of DXA scan.

- Non-ambulatory.

- Functional limitation.

DXA, dual-energy X-ray absorptiometry; NICE, National Institute for Health and Care Excellence; RYGB, Roux-en-Y gastric bypass; SG, sleeve gastrectomy.

attend the study site for monitoring of nutritional intake, vitamin and mineral deficiencies, comorbidities and medication review. Participants will receive verbal PA and dietary advice from a specialist nurse and dietitian at weeks 12 and 36 postsurgery, respectively.

\section{Outcomes measures}

Outcome measures will be collected at four study time points, designed to coincide with normal follow-up care visits; baseline visit at approximately 6 weeks before surgery (T0) then at 3 (T1), 6 (T2) and 12 months (T3) postsurgery (table 1 ).

Sociodemographic, medical history and physical examination Participants' sociodemographic data, medical history and physical examination will be completed by the bariatric team at the baseline visit. Data to be captured including age, gender, ethnicity, educational level, marital status, medication intake, weight history, pregnancy history, alcohol consumption using the Alcohol Use Disorders Identification Test-C (AUDIT-C) questions, ${ }^{42}$ smoking habits and family history of obesity and comorbidities.

\section{Primary outcome}

Body weight will be measured using a weighing scale (Model VT200/220; Vishay Transducers, California, USA) with participants wearing light clothes and without shoes and heavy accessories, to the nearest $0.1 \mathrm{~kg}$. Similarly, height will be determined using a stadiometer (Seca 242, Seca, Hamburg, Germany) to the nearest $0.01 \mathrm{~m}$. \%WL will be calculated using the following formula: $\% \mathrm{WL}=$ ( (weight on the day of surgery-weight at time point after

Table 1 Study timeline and investigations

\begin{tabular}{lcccc}
\hline & Baseline (T0) & $\begin{array}{l}\text { Day of } \\
\text { surgery }\end{array}$ & $\begin{array}{l}\text { 3 months } \\
\text { postsurgery (T1) }\end{array}$ & $\begin{array}{l}\mathbf{6} \text { months } \\
\text { postsurgery (T2) }\end{array}$ \\
\hline postsurgery (T3)
\end{tabular}


surgery) /weight on the day of surgery) $\times 100$, measured at each study time point.

\section{Secondary outcomes}

Body composition (body FM, LMM and BMD)

Body composition will be assessed at baseline and 12 months postsurgery using DXA scan (Discovery A DXA system, software V.13.4.2; Hologic; Massachusetts, USA). DXA scan uses ionising radiation to measure different body compartments. This is the current reference standard for assessing body composition and a gold standard method to diagnose osteopenia and osteoporosis. ${ }^{43}$ In addition, body composition will be measured using BIA (Tanita DC-430MAS; Tanita, Tokyo, Japan) at each study visit. This is a non-invasive, easy to perform and cheaper option to measure body composition that is based on the differences in electrical conductivity of FM and FFM tissues. $^{44}$

\section{PA levels and sedentary behaviour}

PA and time spent in light, moderate and vigorous activities, and sedentary behaviour will be measured objectively using ActiGraph wGT3X-BT (Pensacola, Florida, USA), an accelerometer-based activity monitor. ${ }^{45}$ Participants will be instructed to wear the ActiGraph on their dominant hip for 1 week, from waking in the morning until going to bed at night, and to remove it only during waterbased activities. Additionally, participants will be asked to keep an activity diary throughout the week, to assist interpretation of data from the device. Both the device and activity diary have to be returned to the investigators for data analysis (ActiLife software V.6.13.3; Pensacola, Florida, USA).

\section{Physical function and strength}

Participants' functional capacity will be assessed using a $6 \mathrm{MWT}$, a self-paced, submaximal assessment of functional capacity used to prescribe appropriate exercise. ${ }^{46}$ Lower body functional capacity and strength will be assessed using the STS test. ${ }^{47}$ Static muscle strength will be assessed using Jamar Hydraulic Hand Dynamometer (Patterson Medical; Illinois, USA) ${ }^{48}$

\section{Dietary intake}

All participants will be required to keep a 3 days food diary (two working days and one weekend day) for 1 week at each study time point. This method has a higher agreement with the 9 days food dairy compared with the food frequency questionnaire ${ }^{49}$ while reducing the burden to patients and thus promoting better compliance for documenting food intake. The completed food diary will be returned to the investigators together with the ActiGraph and activity diary by using a stamped addressed envelope provided to participants.

Health-related quality of life

HRQoL will be assessed using EQ-5D-3L and IWQOLLite. The EQ-5D-3L descriptive system is a 5-item self-report questionnaire that assesses the following domains: mobility, self-care, usual activities, pain/discomfort and anxiety/depression, and a visual analogue scale, which records self-rated health on a $0-100$ scale. ${ }^{50}$ EQ-5D-3L health states will be converted into utility values using a formula that attaches weights to each level in each dimension based on valuations by general population samples. We will use a value set for the UK population to calculate utility values at each time point for every participant. ${ }^{51}$ The IWQOL-Lite is a 31-item, self-report, obesity and overweight-specific measure of HRQoL. ${ }^{52}$ This tool consists of a total score and scores on each of five scales physical function, self-esteem, sexual life, public distress, and work; higher scores indicate better HRQoL.

\section{Attitude and symptoms of depression}

BDI-II is a 21-item self-report questionnaire that assesses mood over the past week. ${ }^{53}$ Symptoms of depression are classified by the total score: minimal, mild, moderate, and severe symptoms.

\section{Obesity-associated comorbidities}

Comorbidities (T2D, dyslipidaemia, hypertension, OSA) and medication review will be carried out at each study time point.

\section{Healthcare resource utilisation and costs}

Resource use data will be collected using an adapted version of the CSRI,$^{54}$ including the costs of bariatric surgery plus presurgery visits, number of contacts with healthcare professionals, visits to specialist clinics, the emergency department, admissions to the hospital, primary care contacts, and medications. Resource use data will be converted into costs using published unit costs. ${ }^{55-57}$ In addition, information regarding support from informal carers, employment status and time off work will be collected. Resource use data will be collected for the previous 6 months at the baseline visit and since participants' last study visit at each postsurgery study time point.

\section{Sample size}

A sample size of 100 patients will be enough to model the primary outcome and the range of secondary outcomes with a reasonable level precision and with regard to the number of patients who are likely to be recruited within the study's time frame. Also, a sample size of 100 patients will be sufficient to estimate the $\% \mathrm{WL}$ at 1 year postsurgery to within $\pm 2.5 \%$ using a $95 \%$ CI. This calculation accounts for a possible drop-out rate of up to $25 \%$ and assumes a conservative estimate for SD of \%WL of $10 \%$. This sample size should also ensure that there are enough data points for linear mixed effects models to be fitted with parameter estimates that have a satisfactory level of precision and where the model fitting algorithm will converge.

\section{Statistical analysis}

The demographic and medical history information collected at baseline shall be presented in a table. 
Categorical variables shall be reported as raw numbers and percentages. Reports of continuous variables shall include mean, median, range and SD.

\section{Primary outcome analysis}

The primary outcome is the \%WL measured longitudinally at baseline and 12 months postsurgery. \%WL will be analysed using a linear mixed effects model over the three postsurgery time points ( 3 months, 6 months and 12 months) after controlling for the baseline body weight measure and height. Model assumptions shall be checked and suitable transforms of the primary outcome variable considered if necessary. In addition, overall percentage change in weight since baseline shall be computed marginally at each of 3,6 and 12 months and displayed graphically.

\section{Secondary outcomes analyses}

Analyses of longitudinal secondary outcomes shall be performed using linear mixed effects regression models, with a normal distribution assumed for continuous outcomes (or a suitable transform of these outcomes). Model parameter estimates together with appropriate 95\% CIs shall be reported. Categorical outcomes (eg, proportions of participants with comorbidities) shall be summarised in tabular form at each time point. Where appropriate (for example, for proportions), estimates and $95 \%$ CIs will be presented. To analyse costs, we will assume the costs measured at baseline for the preceding 6 months would persist during follow-up in the absence of surgery; we will then compare postsurgery costs with predicted costs that would have been incurred in the absence of surgery. To account for skewness of the cost data, we will use a generalised linear model with gamma family and log link. ${ }^{58}$

\section{Missing data}

Bias due to missing data will be investigated by comparing the baseline characteristics of participants with and without missing values. Depending on the extent of missingness, the predictors of missing values will be identified. The primary outcome analysis will be adjusted for those predictors of missing values, which are related to missingness. Multiple imputation using chained equations shall be considered as part of a sensitivity analysis for missing data in the primary outcome model.

\section{Data storage and retention}

All data will be handled in accordance with the UK Data Protection Act 1998. Physical data will be stored in a secure room with limited access to only members of the research team, whereas computers storing electronic data will be encrypted and password protected. Each participant will be given a unique study identification number and used on their records instead of their name. The master list linking participants' name and the study identification number will be kept in a secure location. This way, participants' personal identity and data collected in the study cannot be linked by anyone outside the study team. This study is registered with the UCL Data Protection (Reference: Z6364106/2017/04/43). At the end of the study, all essential documentation will be archived securely for a minimum of 20 years from the declaration of the end of study.

\section{Ethics and dissemination}

Potential participants will be explained in detail regarding the aims, methods, anticipated advantages and disadvantages of participation in the study by Good Clinical Practice trained investigators prior to obtaining their written informed consent. Participants will be informed that their participation is on a voluntary basis, and they have the right to withdraw from the study at any time without affecting their present and future medical care. No research procedures will be undertaken prior to patients giving written informed consent. As a duty of care, all possible adverse events will be collected from the day participants consented for the study to monitor their safety.

The findings will be presented to stakeholder groups locally, nationally and internationally and published in peer-reviewed medical journals. The lay-person summary of the findings will be published on the Centre for Obesity Research, UCL website (http://www.ucl.ac.uk/obesity). The results will be fully anonymised, and none of the participants will be identified in any report or publication.

\section{ADVANTAGE AND LIMITATION}

This observational study will address the need for more high-quality data that examine the outcomes of RYGB and SG derived from the UK bariatric population. It will involve a comprehensive assessment and data collection at four study time points in the first year of surgery enabling an indepth analysis of changes in body composition, PA levels, sedentary behaviour, physical function and strength, dietary intake, HRQoL and costs, relative to presurgery. Data collection will be carried out by using validated assessment methods and questionnaires. Another advantage of this study is the use of DXA scan, a reference standard to measure body composition. ${ }^{43}$ Also, the use of accelerometer will generate high-quality data to measure objective PA levels and sedentary behaviour. Studies have shown that bariatric surgery patients tend to over-report their PA levels when assessed using the conventional PA questionnaires. ${ }^{26}$

This protocol for an observational study is not without limitations. First, the study design does not include a conventional intensive lifestyle intervention (non-surgical) as a comparator group. Second, patients will not be randomised for surgical procedure as this study does not aim to compare between RYGB and SG but aims to examine 'real-world' clinical outcomes where the patient/healthcare professional make an informed choice about which procedures is best. However, data that will be generated from this study will allow us to power a subsequent randomised study. Third, a potential sample 
selection bias due to the exclusion of patients with functional limitation (eg, cognitive impairment, walking difficulties) and/or non-ambulatory and patients with more than $200 \mathrm{~kg}$ of body weight owing to the weight limit of the DXA scan. Finally, given resource limitations, only approximately 100 patients will be recruited in this 1-year observational cohort study. Nevertheless, this sample size is adequate to generate indepth insights into the various outcomes of RYGB and SG.

\section{CONCLUSION}

BARI-LIFESTYLE observational study will produce a comprehensive data on the broad range of RYGB and SG outcomes derived from the UK bariatric population that is still scarce in the literature. The information gained from this study will inform future lifestyle programmes for postbariatric surgery patients.

\author{
Author affiliations \\ ${ }^{1}$ Centre for Obesity Research, Division of Medicine, University College London, \\ London, UK \\ ${ }^{2}$ Bariatric Centre for Weight Management and Metabolic Surgery, University College \\ London Hospitals, London, UK \\ ${ }^{3}$ Biomedical Research Centre, National Institute of Health Research University \\ College London Hospitals, London, UK \\ ${ }^{4}$ Institute of Sport, Exercise and Health, London, UK \\ ${ }^{5}$ Department of Statistical Science, University College London, London, UK \\ ${ }^{6}$ Department of Applied Health Research, University College London, London, UK \\ ${ }^{7}$ The Hatter Cardiovascular Institute, Institute of Cardiovascular Science, University \\ College London, London, UK
}

Acknowledgements The authors wish to thank Professor Rumana Omar for her input with regard to the statistical analysis plan. We gratefully acknowledge our Patient and Public Involvement group for their contribution to the study design as to ensure participants' acceptability. We would also like to thank all members of the Steering Committee and our research team at the Centre for Obesity Research, UCL for their invaluable inputs in the study.

Contributors RLB and FCJ designed the overall study and drafted the manuscript. AC coordinated the study to ensure GCP compliance. HK, JD and AK planned the assessment for dietary intake and HRQDL. BP and NL planned the assessment for PA levels, sedentary behaviour and physical function and strength. AG0 advised on the statistical analysis plan. SM contributed to the analysis plan for healthcare resource usage and costs. AP and KC planned the assessment for body composition and review of comorbidities. RLB is the grant holder and chief investigator for the study. All authors have contributed to the refinement of the study protocol and editing the manuscript. All authors have read and approved the final manuscript.

Funding This study is supported by National Institute for Health Research (NIHR), the Sir Jules Thorn Charitable Trust, the Rosetrees Trust, the Stoneygate Trust and Robert Luff Foundation.

Disclaimer The funders were not involved in decisions relating to the study design and data collection. They will not have any role in the study execution, analyses, interpretation of data or writing of the manuscript and decision to submit results.

Competing interests None declared.

Patient consent Not required.

Ethics approval London-Dulwich Research Ethics Committee (Reference: 17/ LO/0950).

Provenance and peer review Not commissioned; externally peer reviewed.

Open Access This is an Open Access article distributed in accordance with the terms of the Creative Commons Attribution (CC BY 4.0) license, which permits others to distribute, remix, adapt and build upon this work, for commercial use, provided the original work is properly cited. See: http://creativecommons.org/ licenses/by/4.0/ (c) Article author(s) (or their employer(s) unless otherwise stated in the text of the article) 2018. All rights reserved. No commercial use is permitted unless otherwise expressly granted.

\section{REFERENCES}

1. NICE. Obesity: Identification, assessment and management of overweight and obesity in children, young people and adults. London: NICE, 2014.

2. Ahmad A, Laverty AA, Aasheim E, et al. Eligibility for bariatric surgery among adults in England: analysis of a national cross-sectional survey. JRSM Open 2014;5:20.

3. Booth HP, Khan O, Fildes A, et al. Changing Epidemiology of Bariatric Surgery in the UK: cohort study using primary care electronic health records. Obes Surg 2016;26:1900-5.

4. Frühbeck G. Bariatric and metabolic surgery: a shift in eligibility and success criteria. Nat Rev Endocrinol 2015;11:465-77.

5. Cummings DE, Cohen RV. Beyond BMI: the need for new guidelines governing the use of bariatric and metabolic surgery. Lancet Diabetes Endocrinol 2014;2:175-81.

6. Schneider J, Peterli R, Gass M, et al. Laparoscopic sleeve gastrectomy and Roux-en-Y gastric bypass lead to equal changes in body composition and energy metabolism 17 months postoperatively: a prospective randomized trial. Surg Obes Relat Dis 2016;12:563-70.

7. Nakamura KM, Haglind EG, Clowes JA, et al. Fracture risk following bariatric surgery: a population-based study. Osteoporos Int 2014;25:151-8.

8. Lu CW, Chang YK, Chang HH, et al. Fracture Risk After Bariatric Surgery: A 12-Year Nationwide Cohort Study. Medicine 2015;94:e2087.

9. Gómez-Ambrosi J, Andrada P, Valentí V, et al. Dissociation of body mass index, excess weight loss and body fat percentage trajectories after 3 years of gastric bypass: relationship with metabolic outcomes. Int J Obes 2017;41:1379-87.

10. Liu C, Wu D, Zhang JF, et al. Changes in Bone Metabolism in Morbidly Obese Patients After Bariatric Surgery: A Meta-Analysis. Obes Surg 2016;26:91-7.

11. Yu EW, Ew Y. Bone metabolism after bariatric surgery. J Bone Miner Res 2014;29:1507-18.

12. Angrisani L, Santonicola A, lovino P, et al. Bariatric Surgery Worldwide 2013. Obes Surg 2015;25:1822-32.

13. Pluskiewicz W, Bužga $M$, Holéczy $P$, et al. Bone mineral changes in spine and proximal femur in individual obese women after laparoscopic sleeve gastrectomy: a short-term study. Obes Surg 2012;22:1068-76.

14. Ruiz-Tovar J, Oller I, Priego P, et al. Short- and mid-term changes in bone mineral density after laparoscopic sleeve gastrectomy. Obes Surg 2013;23:861-6.

15. Carrasco F, Basfi-Fer K, Rojas P, et al. Changes in bone mineral density after sleeve gastrectomy or gastric bypass: relationships with variations in vitamin D, ghrelin, and adiponectin levels. Obes Surg 2014;24:877-84

16. Mechanick JI, Youdim A, Jones DB, et al. Clinical practice guidelines for the perioperative nutritional, metabolic, and nonsurgical support of the bariatric surgery patient-2013 update: cosponsored by American Association of Clinical Endocrinologists, the Obesity Society, and American Society for Metabolic \& Bariatric Surgery. Endocr Pract 2013;19:337-72.

17. Sheets CS, Peat CM, Berg KC, et al. Post-operative psychosocial predictors of outcome in bariatric surgery. Obes Surg 2015;25:330-45.

18. Sarwer DB, Wadden TA, Moore RH, et al. Preoperative eating behavior, postoperative dietary adherence, and weight loss after gastric bypass surgery. Surg Obes Relat Dis 2008;4:640-6.

19. Herman KM, Carver TE, Christou NV, et al. Keeping the weight off: physical activity, sitting time, and weight loss maintenance in bariatric surgery patients 2 to 16 years postsurgery. Obes Surg 2014;24:1064-72.

20. Bond DS, Phelan S, Wolfe LG, et al. Becoming physically active after bariatric surgery is associated with improved weight loss and healthrelated quality of life. Obesity 2009;17:78-83.

21. Campanha-Versiani L, Pereira DA, Ribeiro-Samora GA, et al. The effect of a muscle weight-bearing and aerobic exercise program on the body composition, muscular strength, biochemical markers, and bone mass of obese patients who have undergone gastric bypass surgery. Obes Surg 2017.

22. Vatier C, Henegar C, Ciangura $C$, et al. Dynamic relations between sedentary behavior, physical activity, and body composition after bariatric surgery. Obes Surg 2012;22:1251-6. 
23. Reid RE, Carver TE, Andersen KM, et al. Physical activity and sedentary behavior in bariatric patients long-term post-surgery. Obes Surg 2015;25:1073-7.

24. Biswas A, Pi O, Faulkner GE, et al. Sedentary time and its association with risk for disease incidence, mortality, and hospitalization in adults: a systematic review and meta-analysis. Annals of internal medicine 2015;162:123-32.

25. O'Donovan G, Blazevich AJ, Boreham C, et al. The ABC of Physical Activity for Health: a consensus statement from the British Association of Sport and Exercise Sciences. J Sports Sci 2010;28:573-91.

26. Herring LY, Stevinson C, Davies MJ, et al. Changes in physical activity behaviour and physical function after bariatric surgery: a systematic review and meta-analysis. Obesity Reviews 2016;17:250-61.

27. Afshar S, Seymour K, Kelly SB, et al. Changes in physical activity after bariatric surgery: using objective and self-reported measures. Surgery for Obesity and Related Diseases 2017;13:474-83.

28. Raftopoulos I, Bernstein B, O'Hara K, et al. Protein intake compliance of morbidly obese patients undergoing bariatric surgery and its effect on weight loss and biochemical parameters. Surgery for Obesity and Related Diseases 2011;7:733-42.

29. Sherf Dagan S, Tovim TB, Keidar A, et al. Inadequate protein intake after laparoscopic sleeve gastrectomy surgery is associated with a greater fat free mass loss. Surgery for Obesity and Related Diseases 2017;13:101-9.

30. Moizé V, Andreu A, Rodríguez L, et al. Protein intake and lean tissue mass retention following bariatric surgery. Clinical Nutrition 2013;32:550-5

31. Faria SL, Faria OP, Buffington C, et al. Dietary protein intake and bariatric surgery patients: a review. Obes Surg 2011;21:1798-805.

32. Ito MK, Gonçalves VSS, Faria SLCM, et al. Effect of Protein Intake on the Protein Status and Lean Mass of Post-Bariatric Surgery Patients: a Systematic Review. Obes Surg 2017;27:502-12.

33. Kolotkin RL, Meter K, Williams GR. Quality of life and obesity. Obesity Reviews 2001;2:219-29.

34. Munoz DJ, Lal M, Chen EY, et al. Why patients seek bariatric surgery: a qualitative and quantitative analysis of patient motivation. Obes Surg 2007;17:1487-91.

35. Testa MA, Simonson DC. Assessment of quality-of-life outcomes. $N$ Engl J Med Overseas Ed 1996;334:835-40.

36. Kolotkin RL, Andersen JR. A systematic review of reviews: exploring the relationship between obesity, weight loss and health-related quality of life. Clin Obes 2017;7:273-89.

37. Lindekilde N, Gladstone BP, Lübeck M, et al. The impact of bariatric surgery on quality of life: a systematic review and meta-analysis. Obesity Reviews 2015;16:639-51.

38. Pollock RF, Chilcott J, Muduma G, et al. Laparoscopic adjustable gastric banding vs standard medical management in obese patients with type 2 diabetes: a budget impact analysis in the UK. J Med Econ 2013;16:249-59.

39. Terranova L, Busetto L, Vestri A, et al. Bariatric surgery: costeffectiveness and budget impact. Obes Surg 2012;22:646-53.
40. Ackroyd R, Mouiel J, Chevallier JM, et al. Cost-effectiveness and budget impact of obesity surgery in patients with type-2 diabetes in three European countries. Obes Surg 2006;16:1488-503.

41. De Luca M, Angrisani L, Himpens J, et al. Indications for surgery for obesity and weight-related diseases: position statements from the International Federation for the Surgery of Obesity and Metabolic Disorders (IFSO). Obes Surg 2016;26:1659-96.

42. Bush K, Kivlahan DR, McDonell MB, et al. The AUDIT alcohol consumption questions (AUDIT-C): an effective brief screening test for problem drinking. Ambulatory Care Quality Improvement Project (ACQUIP). Alcohol use disorders identification test. Archives of internal medicine 1998;158:1789-95.

43. Lee SY, Gallagher D. Assessment methods in human body composition. Curr Opin Clin Nutr Metab Care 2008;11:566-72.

44. Faria SL, Faria OP, Cardeal MD, et al. Validation study of multi-frequency bioelectrical impedance with dual-energy X-ray absorptiometry among obese patients. Obes Surg 2014;24:1476-80.

45. Migueles $\mathrm{JH}$, Cadenas-Sanchez $\mathrm{C}$, Ekelund U, et al. Accelerometer data collection and processing criteria to assess physical activity and other outcomes: a systematic review and practical considerations. Sports Medicine 2017;47:1821-45.

46. ATS Committee on Proficiency Standards for Clinical Pulmonary Function Laboratories. ATS statement: guidelines for the six-minute walk test. Am J Respir Crit Care Med 2002;166:111-7.

47. Pataky Z, Armand S, Müller-Pinget S, et al. Effects of obesity on functional capacity. Obesity 2014;22:56-62.

48. Sousa-Santos AR, Amaral TF. Differences in handgrip strength protocols to identify sarcopenia and frailty - a systematic review. BMC Geriatr 2017;17:238.

49. Yang YJ, Kim MK, Hwang SH, et al. Relative validities of 3-day food records and the food frequency questionnaire. Nutr Res Pract 2010;4:142-8.

50. Brooks R. EuroQol: the current state of play. Health Policy 1996;37:53-72.

51. Dolan P. Modeling valuations for EuroQol health states. Med Care 1997;35:1095-108.

52. Kolotkin RL, Crosby RD, Kosloski KD, et al. Development of a brief measure to assess quality of life in obesity. Obes Res 2001;9:102-11.

53. Beck AT, Steer RA, Brown GK. Manual for the Beck Depression Inventory-II. San Antonio, TX, 1996.

54. Beecham J, Knapp M. Costing psychiatric interventions. In: Thornicroft G, ed. Measuring Mental Health Needs. 2nf edition: Gaskell, 2001:200-24.

55. Curtis L, Burns A. Unit Costs of Health and Social Care 2015. Canterbury: Personal Social Services Research Unit, University of Kent, 2015.

56. Department of Health. National Schedule of Reference Costs. London: Department of Health, 2015.

57. British National Formulary. Authoritative and practical. 2018. https:// www.bnf.org/

58. Barber J, Thompson S. Multiple regression of cost data: use of generalised linear models. J Health Serv Res Policy 2004;9:197-204. 